UH-511-992-01

October 2001

\title{
CPT AND LORENTZ VIOLATIONS IN NEUTRINO OSCILLATIONS $^{a}$
}

\author{
SANDIP PAKVASA \\ Department of Physics and Astronomy, \\ University of Hawaii, \\ Honolulu, HI 96822, USA \\ Email: pakvasa@phys.hawaii.edu
}

\begin{abstract}
Tests of Lorentz invariance violation and CPT Violation in neutrino oscillations are discussed. The sensitivity of current and future experiments is presented.
\end{abstract}

\section{Introduction}

We have heard about how we expect both CPT and Lorentz symmetries to be violated at very small scalest Neutrino oscillations are surprisingly sensitive to CPT and Lorentz violations and very strong constraints can be placed on symmetry breaking parameters. I will review the current status.

\section{CPT Violation in Neutrino Oscillations ${ }^{2}$}

Consequences of $C P, T$ and $C P T$ violation for neutrino oscillations have been written down before 3 . We summarize them briefly for the $\nu_{\alpha} \rightarrow \nu_{\beta}$ flavor oscillation probabilities $P_{\alpha \beta}$ at a distance $L$ from the source. If

$$
P_{\alpha \beta}(L) \neq P_{\bar{\alpha} \bar{\beta}}(L), \quad \beta \neq \alpha,
$$

then $C P$ is not conserved. If

$$
P_{\alpha \beta}(L) \neq P_{\beta \alpha}(L), \quad \beta \neq \alpha,
$$

then $T$-invariance is violated. If

$$
P_{\alpha \beta}(L) \neq P_{\bar{\beta} \bar{\alpha}}(L), \quad \beta \neq \alpha,
$$

or

${ }^{a}$ Presented at the Second Meeting on CPT and Lorentz Symmetry CPT'01, August 15-18, 2001, Bloomington, Indiana. 


$$
P_{\alpha \alpha}(L) \neq P_{\bar{\alpha} \bar{\alpha}}(L)
$$

then $C P T$ is violated. When neutrinos propagate in matter, matter effects give rise to apparent $C P$ and $C P T$ violation even if the mass matrix is $C P$ conserving.

The $C P T$ violating terms can be Lorentz-invariance violating (LV) or Lorentz invariant. The Lorentz-invariance violating, $C P T$ violating case has been discussed by Colladay and Kosteleckyt and by Coleman and Glashow 0 .

The effective LV CPT violating interaction for neutrinos is of the form

$$
\bar{\nu}_{L}^{\alpha} b_{\mu}^{\alpha \beta} \gamma_{\mu} \nu_{L}^{\beta},
$$

where $\alpha$ and $\beta$ are flavor indices. We assume rotational invariance in the "preferred" frame, in which the cosmicmicrowave background radiation is isotropic

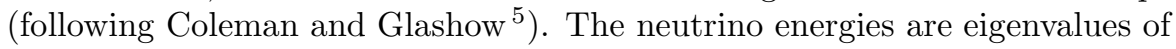

$$
m^{2} / 2 p+b_{0},
$$

where $b_{0}$ is a hermitian matrix, hereafter labeled $b$. Consequences of the rotational invariance violating terms $\underline{b}$ are being investigated 6 .

In the two-flavor case the neutrino phases may be chosen such that $b$ is real, in which case the interaction in Eq. (5) is $C P T$ odd The survival probabilities for flavors $\alpha$ and $\bar{\alpha}$ produced at $t=0$ are given by

$$
P_{\alpha \alpha}(L)=1-\sin ^{2} 2 \Theta \sin ^{2}(\Delta L / 4),
$$

and

$$
P_{\bar{\alpha} \bar{\alpha}}(L)=1-\sin ^{2} 2 \bar{\Theta} \sin ^{2}(\bar{\Delta} L / 4),
$$

where

$$
\begin{aligned}
\Delta \sin 2 \Theta & =\left|\left(\delta m^{2} / E\right) \sin 2 \theta_{m}+2 \delta b e^{i \eta} \sin 2 \theta_{b}\right|, \\
\Delta \cos 2 \Theta & =\left(\delta m^{2} / E\right) \cos 2 \theta_{m}+2 \delta b \cos 2 \theta_{b} .
\end{aligned}
$$

$\bar{\Delta}$ and $\bar{\Theta}$ are defined by similar equations with $\delta b \rightarrow-\delta b$. Here $\theta_{m}$ and $\theta_{b}$ define the rotation angles that diagonalize $m^{2}$ and $b$, respectively, $\delta m^{2}=m_{2}^{2}-m_{1}^{2}$ and $\delta b=b_{2}-b_{1}$, where $m_{i}^{2}$ and $b_{i}$ are the respective eigenvalues. We use the convention that $\cos 2 \theta_{m}$ and $\cos 2 \theta_{b}$ are positive and that $\delta m^{2}$ and $\delta b$ can have either sign. The phase $\eta$ in Eq. (9) is the difference of the phases in the unitary matrices that diagonalize $\delta m^{2}$ and $\delta b$; only one of these two phases can be absorbed by a redefinition of the neutrino states.

Observable $C P T$-violation in the two-flavor case is a consequence of the interference of the $\delta m^{2}$ terms (which are $C P T$-even) and the LV terms in Eq. (5) (which are $C P T$-odd); if $\delta m^{2}=0$ or $\delta b=0$, then there is no observable 
$C P T$-violating effect in neutrino oscillations. If $\delta m^{2} / E \gg 2 \delta b$ then $\Theta \simeq \theta_{m}$ and $\Delta \simeq \delta m^{2} / E$, whereas if $\delta m^{2} / E \ll 2 \delta b$ then $\Theta \simeq \theta_{b}$ and $\Delta \simeq 2 \delta b$. Hence the effective mixing angle and oscillation wavelength can vary dramatically with $E$ for appropriate values of $\delta b$.

We note that a $C P T$-odd resonance for neutrinos $\left(\sin ^{2} 2 \Theta=1\right)$ occurs whenever $\cos 2 \Theta=0$ or

$$
\left(\delta m^{2} / E\right) \cos 2 \theta_{m}+2 \delta b \cos 2 \theta_{b}=0 ;
$$

similar to the resonance due to matter effects 6 . The condition for antineutrinos is the same except $\delta b$ is replaced by $-\delta b$. The resonance occurs for neutrinos if $\delta m^{2}$ and $\delta b$ have the opposite sign, and for antineutrinos if they have the same sign. A resonance can occur even when $\theta_{m}$ and $\theta_{b}$ are both small, and for all values of $\eta$; if $\theta_{m}=\theta_{b}$, a resonance can occur only if $\eta \neq 0$. If one of $\nu_{\alpha}$ or $\nu_{\beta}$ is $\nu_{e}$, then matter effects have to be included.

If $\eta=0$, then

$$
\begin{aligned}
\Theta & =\theta, \\
\Delta & =\left(\delta m^{2} / E\right)+2 \delta b .
\end{aligned}
$$

In this case a resonance is not possible. The oscillation probabilities become

$$
\begin{aligned}
& P_{\alpha \alpha}(L)=1-\sin ^{2} 2 \theta \sin ^{2}\left\{\left(\frac{\delta m^{2}}{4 E}+\frac{\delta b}{2}\right) L\right\}, \\
& P_{\bar{\alpha} \bar{\alpha}}(L)=1-\sin ^{2} 2 \theta \sin ^{2}\left\{\left(\frac{\delta m^{2}}{4 E}-\frac{\delta b}{2}\right) L\right\} .
\end{aligned}
$$

For fixed $E$, the $\delta b$ terms act as a phase shift in the oscillation argument; for fixed $L$, the $\delta b$ terms act as a modification of the oscillation wavelength.

An approximate direct limit on $\delta b$ when $\alpha=\mu$ can be obtained by noting that in atmospheric neutrino data the flux ofdownward going $\nu_{\mu}$ is not depleted whereas that of upward going $\nu_{\mu}$ is depleted 6 . Hence, the oscillation arguments in Eqs. (14) and (15) cannot have fully developed for downward neutrinos. Taking $|\delta b L / 2|<\pi / 2$ with $L \sim 20 \mathrm{~km}$ for downward events leads to the upper bound $|\delta b|<3 \times 10^{-20} \mathrm{GeV}$; the $\mathrm{K} 2 \mathrm{~K}$ results can improve this by an order of magnitude; upward going events could in principle test $|\delta b|$ as low as $5 \times 10^{-23} \mathrm{GeV}$. Since the $C P T$-odd oscillation argument depends on $L$ and the ordinary oscillation argument on $L / E$, improved direct limits could be obtained by a dedicated study of the energy and zenith angle dependence of the atmospheric neutrino data. 
The difference between $P_{\alpha \alpha}$ and $P_{\bar{\alpha} \bar{\alpha}}$

$$
P_{\alpha \alpha}(L)-P_{\bar{\alpha} \bar{\alpha}}(L)=-2 \sin ^{2} 2 \theta \sin \left(\frac{\delta m^{2} L}{2 E}\right) \sin (\delta b L),
$$

can be used to test for $C P T$-violation. In a neutrino factory, the ratio of $\bar{\nu}_{\mu} \rightarrow \bar{\nu}_{\mu}$ to $\nu_{\mu} \rightarrow \nu_{\mu}$ events will differ from the standard model (or any local quantum field theory model) value if $C P T$ is violated. Fig. 1 shows the event ratios $N\left(\bar{\nu}_{\mu} \rightarrow \bar{\nu}_{\mu}\right) / N\left(\nu_{\mu} \rightarrow \nu_{\mu}\right)$ versus $\delta b$ for a neutrino factory with $10^{19}$ stored muons and a $10 \mathrm{kt}$ detector at several values of stored muon energy, assuming $\delta m^{2}=3.5 \times 10^{-3} \mathrm{eV}^{2}$ and $\sin ^{2} 2 \theta=1$, as indicated by the atmospheric neutrino data 9 . The lack of equality of $\nu$ and $\bar{\nu}$ events at $\delta b=0$ comes about due to the difference between $\nu$ and $\bar{\nu}$ cross-sections, assuming equal fluxes. The error bars in Fig. 1 are representative statistical uncertainties. The node near $\delta b=8 \times 10^{-22} \mathrm{GeV}$ is a consequence of the fact that $P_{\alpha \alpha}=P_{\bar{\alpha} \bar{\alpha}}$, independent of $E$, whenever $\delta b L=n \pi$, where $n$ is any integer; the node in Fig. 1 is for $n=1$. A $3 \sigma C P T$ violation effect is possible in such an experiment for $\delta b$ as low as $3 \times 10^{-23} \mathrm{GeV}$ for stored muon energies of $20 \mathrm{GeV}$. For $\nu_{e}^{\prime} s$, if the relevant $\delta m^{2}$ is smaller than $10^{-10} \mathrm{eV}^{2}$ ("just-so"), then for large mixing $\delta b_{\nu_{e} \nu_{x}}$ can be bounded by $10^{-27} \mathrm{GeV}$ from solar neutrino data. If all $\delta m^{2}$ 's were sufficiently small $\left(<10^{-17} \mathrm{eV}^{2}\right)$, then data from SN1987A and future data from AGN neutrinos can probe $\delta b$ down to $10^{-40} \mathrm{GeV}$.

We have also checked 3 the observability of $C P T$ violation at other distances, assuming the same neutrino factory parameters used above. For $L=$ $250 \mathrm{~km}$, the $\delta b L$ oscillation argument in Eq. (16) has not fully developed and the ratio of $\bar{\nu}$ to $\nu$ events is still relatively close to the standard model value. For $L=2900 \mathrm{~km}$, a $\delta b$ as low as $10^{-23} \mathrm{GeV}$ may be observable at the $3 \sigma$ level. However, longer distances also have matter effects that simulate $C P T$ violation, which have to be corrected for.

Lorentz invariant CPT violation can arise if e.g. $\delta m_{i j}^{2}$ and $\theta_{i j}$ are different for nejtrinos and antineutrinos. Constraints on such differences are rather weak 2 . Taking advantage of this, a very intriguing proposal has been made by Barenboim et al.10 They propose that in the $\nu$ sector, the $\delta m^{2}$ and mixing are "conventional" and nearly bimaximal; namely $\delta m_{23}^{2} \sim$ atmospheric and $\delta m_{21}^{2} \sim$ LMA. Whereas in the $\bar{\nu}$ sector $\delta m_{23}^{2} \sim 0\left(e V^{2}\right), \delta m_{21}^{2} \sim$ atmospheric and the mixing is large in the 1-2 sector and small (of order LSND) in 2-3 sector. Then the $\overline{\nu_{\mu}}-\bar{\nu}_{e}$ conversion in LSND11 is accounted for, and the solar neutrinos are unaffected as no $\bar{\nu}^{\prime} s$ are emitted in the sun. This proposal can be tested by Mini-Boone seeing LSND effect in $\bar{\nu}_{\mu}$ beam but not in the $\nu_{\mu}$ beam and by the fact that the $\nu_{e}$ and $\bar{\nu}_{e}$ oscillations with $\delta m_{\text {atm }}^{2}$ will be very different (present in former and absent in latter). For example, KAMLAND 12 
will see no effect in Reactor $\bar{\nu}_{e}^{\prime} s$ even if LMA is the correct solution for solar $\nu_{e}^{\prime} s$.

\section{Lorentz Invariance Violation in Neutrino Oscillations}

A general formalism to describe small departures from exact Lorentz invariance has been developed by Colladay and Kostelecky 13 . This modification of Standard Model is renormalizable and preserves the gauge symmetries. When rotational invariance in a preferred frame is imposed, the formalism developed by Coleman and Glashow 14 can be used. In this form, the main effect (at high energies) of the violation of Lorentz invariance is that each particle species $i$ has its own maximum attainable velocity $(\mathrm{MAV}), c_{i}$, in this frame. The Lorentz violating parameters are $c_{i}^{2}-c_{j}^{2}$.

There are many interesting consequences 14 : evading of GZK cut-off, possibility of "forbidden" processes at high thresholds e.g. $\gamma \rightarrow e^{+}+e^{-}, p \rightarrow$ $e^{+}+n+\nu, \mu \rightarrow \pi+\nu_{\mu}, \mu \rightarrow e+\gamma$ etc. Another new allowed phenomenon is the oscillation of massless neutrinos.

Even if neutrinos were massless, the flavor eigenstates could be mixtures of velocity (MAV) eigenstates and the flavor survival probability (in the two flavor case) is given by

$$
P_{\alpha \alpha}=1-\sin ^{2} 2 \theta \sin ^{2}\left(\frac{\delta c}{2} L E\right)
$$

where $\delta c=c_{1}-c_{2}$.

Identical phenomenology for neutrino oscillations arises in the case of flavor violating gravity or the violation of equivalence principle, with $\delta \gamma \Phi$ replacing $\delta c$. Here, $\delta \gamma=\gamma_{1}-\gamma_{2}$ is the difference in the PPN parameters which violates the equivalence principle and $\Phi$ is the gravitational potential. This test of equivalence principle was first proposed 15 by Gasperini and by Halprin and Leung. There does not seem to be a consistent theoretical scheme from which such consequences follow. In other words, a theory of gravity which would agree with the classic tests of GR and also violate equivalence principle has not yet been found 16 .

This form of massless neutrino oscillations was very interesting at one time. The reason was that a single choice of parameters $\delta c$ and $\sin ^{2} 2 \theta$ cguld account for both atmospheric and solar neutrinos with $\nu_{e}-\nu_{\mu}$ mixing 17. However, now $\nu_{\mu}-\nu_{e}$ can no longer account for atmosphericneutrinos 18 and the LE dependence is ruled out for atmospheric neutrinos 19 . A description of solar neutrinos, even including the recent SNO data, is still possible 20 ; with the choice of parameters: $\delta c / 2 \sim 10^{-24}$ and large mixing. For $\nu_{\mu}-\nu_{x}$ mixing, 
the results of NUTEV21 can be used to constrain $\delta c / 2<10^{-21}$ (for $\sin ^{2} 2 \theta>$ $10^{-3}$ ), and future Long Baseline experiments 22 will extend the bounds to $10^{-23}$ for large mixing. by

In the general case, when neutrinos are not massless, the energies are given

$$
E_{i}=p+m_{i}^{2} / 2 p+c_{i} p
$$

There will be two mixing angles (even for two flavors) and the survival probability is given by

$$
P_{\alpha \alpha}=1-\sin ^{2} 2 \Theta \sin ^{2}(\Delta L / 4)
$$

where

$$
\begin{aligned}
\Delta \sin 2 \Theta & =\left|\left(\delta m^{2} / E\right) \sin 2 \theta_{m}+2 \delta c e^{i \eta} E \sin 2 \theta_{c}\right|, \\
\Delta \cos 2 \Theta & =\left(\delta m^{2} / E\right) \cos 2 \theta_{m}+2 \delta c E \cos 2 \theta_{c}
\end{aligned}
$$

One can also write the most general expression including the CPT violating term of Eq. (6) and even extending to three flavors. But there is not enough information to constraint the many new parameters.

\section{Summary}

When data from Long Baseline experiments and eventually neutrino factories become available, CPT and Lorentz violation in neutrino oscillations can be probed to new and significant levels. It would be especially useful to have detectors capable of distinguishing between $\nu$ and $\bar{\nu}$ events.

\section{Acknowledgments}

I thank Vernon Barger, Alan Kostelecky and Tom Weiler for discussions. This work is supported in part by U.S.D.O.E. under grant DE-FG 03-94ER40833.

\section{References}

1. See talks by J. Pullin, A. Kostelecky, F. Mansouri, R. Potting, G. Amelino-Camelia and D. Colladay, in these Proceedings.

2. V. Barger, S. Pakvasa, T.J. Weiler, and K. Whisnant, Phys. Rev. Lett. 85, 5055 (2000).

3. N. Cabibbo, Phys. Lett. B72, 333 (1978); V. Barger, K. Whisnant, R.J.N. Phillips, Phys. Rev. Lett. 45, 2084 (1980); S. Pakvasa, in Proc. of the XXth International Conference on High Energy Physics, ed. by L. Durand and L.G. Pondrom, AIP Conf. Proc. No. 68 (AIP, New York, 1981), Vol. 2, p. 1164. 
4. D. Colladay and V.A. Kostelecky, Phys. Rev. D55, 6760 (1997).

5. S. Coleman and S.L. Glashow, Phys. Rev. D59, 116008 (1999).

6. A. Kostelecky, S. Pakvasa and T.J. Weiler (work in progress).

7. V. Barger, K. Whisnant, S. Pakvasa, and R.J.N. Phillips, Phys. Rev. D22, 2718 (1980).

8. L. Wolfenstein, Phys. Rev. D17, 2369 (1978); S.P. Mikheyev and A. Smirnov, Yad. Fiz. 42, 1441 (1985) [Sov. J. Nucl. Phys. 42, 913 (1986)].

9. Kamiokande collaboration, K.S. Hirata et al., Phys. Lett. B280, 146 (1992); Y. Fukuda et al., Phys. Lett. B335, 237 (1994); IMB collaboration, R. Becker-Szendy et al., Nucl. Phys. Proc. Suppl. 38B, 331 (1995); Soudan-2 collaboration, W.W.M. Allison et al., Phys. Lett. B391, 491 (1997); MACRO collaboration, M. Ambrosio et al., Phys. Lett. B434, 451 (1998); Super-Kamiokande Collaboration, Y. Fukuda et al., Phys. Rev. Lett. 81, 1562 (1998); Phys. Rev. Lett. 82, 2644 (1999).

10. G. Barenboim, L. Borisov, J. Lykken and A. Yu Smirnov, hepph/0108199; for a similar earlier suggestion see H. Murayama and T. Yanagida, hep-ph/0010178.

11. A. Aguilar et al. hep-ex/010409.

12. A. Suzuki, 8th International Workshop on Neutrino Telescopes, Venice, Feb. 23-26, 1999, Ed. M. Baldo-Ceolin, Vol. I. p. 325.

13. D. Colladay and A. Kostelecky, Phys. Rev. D58, 116002 (1998).

14. S. Coleman and S.L. Glashow, Phys. Lett. B405, 249 (1997).

15. M. Gasperini, Phys. Rev. D38, 2635 (1988), A. Halprin and C.N. Leung, Phys. Rev. Lett. 67, 1833 (1991).

16. A. Halprin, C.N. Leung and J. Pantaleone, Phys. Rev D53, 5365 (1996).

17. S. Glashow et al, Phys. Rev. D56, 2433 (1997); A. Halprin, C.N. Leung and J. Pantaleone, Phys. Rev. D47, 4199 (1993). J.N Bahcall, P. Krastev and C.N. Leung, Phys. Rev. D52, 1770 (1996); H. Minakata and H. Nunokawa, Phys. Rev. D51, 6625 (1995).

18. The Super-Kamiokande Collaboration, Phys. Rev. Lett. 81, 1562 (1998).

19. J.G. Learned, Current Aspects of Neutrino Physics, Ed. D. Caldwell, Springer-Verlag (2001), hep-ex/0107056; P. Lipari and M. Lusignoli, Phys. Rev. D60, 0113003 (1999); G.L. Fogli, E. Lisi and A. Marrone, Phys. Rev. D60, 053006 (1999).

20. A. Raychaudhuri and A. Sil, hep-ph/0107022; A. M. Gago, H. Nunokawa and R. Zukanovich-Funchal, Phys. Rev. Lett. 84, 2035 (2000).

21. A. Romosan et al, Phys. Rev. D59, 031101 (1999); Phys. Rev. Lett. 78, 2912 (1997).

22. MINOS Collaboration, NUMI-2-375 Report (1998); NGS Report, CERN 
98-02, INFN/AE-98-05 (1998); for the range that can be probed at neutrino factories, see A. Datta, Phys. Lett. B504, 247 (2001). 


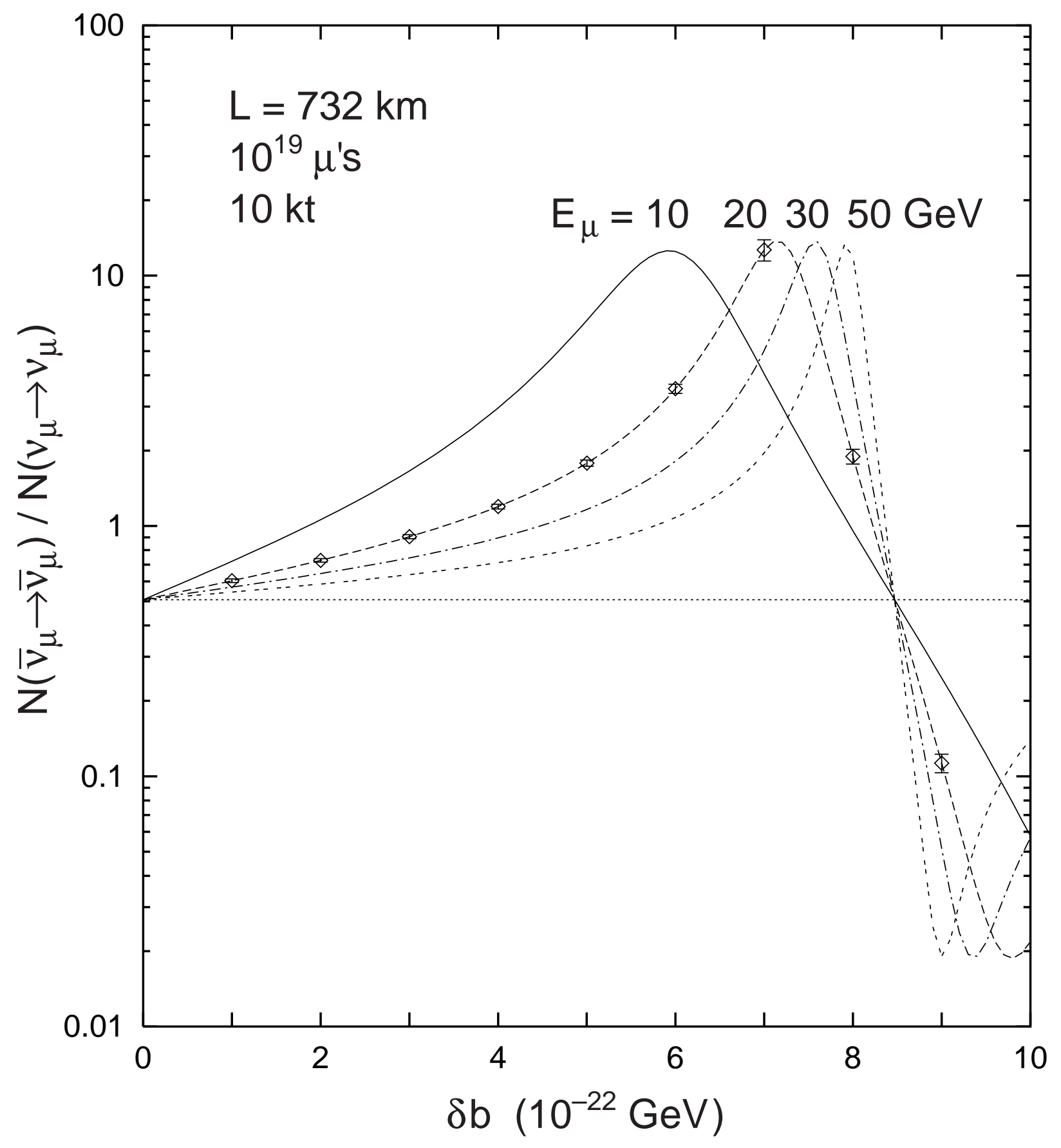

